\title{
OPEN Osteoprotegerin is a marker of cardiovascular mortality in patients with chronic kidney disease stages 3-5
}

\author{
Gustavo Lenci Marques ${ }^{1 \bowtie}$, Shirley Hayashi ${ }^{2}$, Anna Bjällmark ${ }^{3}$, Matilda Larsson ${ }^{4}$, \\ Miguel Riella ${ }^{5}$, Marcia Olandoski ${ }^{6}$, Bengt Lindholm² \& Marcelo Mazza Nascimento ${ }^{1,2}$
}

Cardiovascular disease (CVD) is the leading cause of death in patients with chronic kidney disease (CKD). Osteoprotegerin (OPG), known to regulate bone mass by inhibiting osteoclast differentiation and activation, might also play a role in vascular calcification. Increased circulating OPG levels in patients with CKD are associated with aortic calcification and increased mortality. We assessed the predictive role of OPG for all-cause and cardiovascular mortality in patients with CKD stages 3-5 over a 5-year follow-up period. We evaluated the relationship between OPG and all-cause and cardiovascular mortality in 145 CKD patients (stages 3-5) in a prospective observational follow-up study. Inflammation markers, including high-sensitivity C-reactive protein, standard echocardiography, and estimation of intima-media thickness in the common carotid artery, were assessed at baseline, and correlations with OPG levels were determined. The cutoff values for OPG were defined using ROC curves for cardiovascular mortality. Survival was assessed during follow up lasting for up to 5.5 years using Fine and Gray model. A total of 145 ( 89 men; age $58.9 \pm 15.0$ years) were followed up. The cutoff value for OPG determined using ROC was $10 \mathrm{pmol} / \mathrm{L}$ for general causes mortality and $10.08 \mathrm{pmol} / \mathrm{L}$ for CV causes mortality. Patients with higher serum OPG levels presented with higher mortality rates compared to patients with lower levels. Aalen-Johansen cumulative incidence curve analysis demonstrated significantly worse survival rates in individuals with higher baseline OPG levels for all-cause and cardiovascular mortality $(p<0.001)$. In multivariate analysis, OPG was a marker of general and cardiovascular mortality independent of sex, age, CVD, diabetes, and CRP levels. When CKD stages were included in the multivariate analysis, OPG was an independent marker of all-cause mortality but not cardiovascular mortality. Elevated serum OPG levels were associated with higher all-cause and cardiovascular mortality risk, independent of age, CVD, diabetes, and inflammatory markers, in patients with CKD.

Cardiovascular disease (CVD) accounts for about $30 \%$ of deaths worldwide ${ }^{1}$ and is the leading cause of death in patients with chronic kidney disease $(\mathrm{CKD})^{2}$. However, the high prevalence of traditional risk factors cannot fully explain the high incidence of fatal events in this population ${ }^{3,4}$. Therefore, in the last decade, other risk factors, such as markers of inflammation, oxidative stress, and vascular calcification, have been studied ${ }^{5-7}$. In this context, osteoprotegerin (OPG) has become the subject of increased interest because of its role as a cardiovascular risk factor in both the general population as well as in CKD patients ${ }^{8}$.

OPG is a soluble receptor of the osteoclast activator, receptor activator of nuclear factor- $\kappa \mathrm{B}$ ligand (RANKL), and has been studied as an important marker of CVD in the pathogenesis of vascular calcification and atherosclerosis ${ }^{9,10}$. Elevated serum OPG levels seem to be associated with increased morbidity and mortality in patients with coronary artery disease and heart failure. A recent meta-analysis demonstrated a significant association between circulating OPG levels and mortality in the general population ${ }^{11}$.

\footnotetext{
${ }^{1}$ Department of Internal Medicine, Hospital de Clínicas, Federal University of Paraná, General Carneiro, 181, Curitiba, PR 80060-900, Brazil. 'Divisions of Renal Medicine and Baxter Novum, Department of Clinical Science, Intervention and Technology, Karolinska Institute, Karolinska University Hospital in Huddinge, Stockholm, Sweden. ${ }^{3}$ School of Health and Welfare, Jönköping University, Jönköping, Sweden. ${ }^{4}$ Royal Institute of Technology, Stockholm, Sweden. ${ }^{5}$ ProRenal Foundation, Curitiba, PR, Brazil. ${ }^{6}$ Pontifícia Universidade Católica Do Paraná, Curitiba, PR, Brazil. ${ }^{\varpi}$ email: gustavolencimarques@gmail.com
} 
OPG has also been associated with higher mortality in patients undergoing hemodialysis ${ }^{12}$. In a preliminary observational study, our research group found a significant association between elevated OPG levels and the presence of increased all-cause mortality in a cohort of patients with CKD stages 3-5 over a 3-year follow-up period $^{13}$. To date, our research group has not studied the association between elevated serum OPG levels and cardiovascular mortality or its interaction with other myocardial injury markers.

We hypothesized a possible correlation between elevated serum OPG levels in patients with CKD stages 3-5 and markers of presence and severity of CVD, and investigated whether OPG is a possible independent marker of cardiovascular mortality in patients with CKD over a 5-year follow-up period.

\section{Materials and methods}

Patients with CKD stages 3-5, including those undergoing dialysis at Pró-Renal Foundation in Curitiba, Brazil, were considered for enrollment in this study. All patients gave written informed consent and the ethics committee of the Hospital Evangelico de Curitiba approved the study protocol and all the study procedures were carried out in accordance with relevant guidelines. The exclusion criteria were dialysis treatment lasting for less than 1 month, age younger than 18 years, the presence of HIV or hepatitis B/C infection, and other chronic inflammatory diseases.

Study design. All patients enrolled through the cohort study design underwent a baseline investigation comprising blood sampling and cardiovascular assessment. They were subsequently followed up for up to 66 months for the analysis of survival. The observation period was from March 2008 to December 2015.

Cardiovascular and laboratory assessment. The intima-media thickness (IMT) was evaluated using semiautomatic edge detection software (GE Vingmed Ultrasound, Horten, Norway) according to the recommendations of the American Society of Echocardiography ${ }^{14}$. All two-dimensional and Doppler variables were acquired and analyzed according to the guidelines of the American Society of Echocardiography ${ }^{15,16}$. All ultrasound examinations were performed only once, when the patient was included in the cohort.

Blood samples were collected from patients after overnight fasting. The samples were collected midweek from hemodialysis patients and at regular clinic visits from other patients with CKD stages 3-5, including those undergoing peritoneal dialysis. All analyses were performed using automated analyzers at the Renal Medicine Laboratory, Clinical Research Center, Karolinska Institutet, Stockholm, Sweden ${ }^{13}$. Serum OPG was measured using a ELISA assay commercial kit (R\&D Systems Inc.). Plasma and serum were stored at $-70{ }^{\circ} \mathrm{C}$ for transport, the analyzes were carried out as soon as the material reached its destination.

Statistical analysis. Data are reported as median (interquartile range) or mean \pm standard deviation (SD), and as frequencies and percentages for categorical variables, as appropriate. For determining cutoff values for OPG associated with cardiovascular death, receiver operating characteristic (ROC) curves were adjusted and the corresponding area under curve (AUC) was evaluated. Best cutoff was determined using Youden index criteria. To compare two groups with continuous quantitative variables, we used a Student's t-test for independent samples. The non-normally distributed variables were log-transformed and parametric tests were applied. We used Fisher's exact test for categorical variables. Three groups were compared using Chi-square test or one-way analysis of variance (ANOVA) and Bonferroni post-hoc test. For analyzing factors associated with the OPG $(\mathrm{pg} / \mathrm{mL})$ level initially, a univariate analysis was performed estimating Spearman's correlation coefficients. Subsequently, a multivariate linear regression model was adjusted, including as explanatory variables those that presented significant correlations with OPG in the univariate analysis. The response variable for multiple linear regression was log-OPG and included log-transformed explanatory variables. Residuals were checked by normal probability plot and predicted values versus residuals scatterplot. Fine and Gray models were adjusted to analyze mortality. For all-cause mortality models, transplantation was considered as the competing risk. For cardiovascular models, other causes of mortality and transplantation were considered as competing risks. The estimated association measure was the subdistribution hazard ratio (SHR) provided for unit change with $95 \%$ confidence interval. Aalen-Johansen cumulative incidence curves were presented for OPG. The selection of variables for multivariate analysis models used clinically relevant predictors. The first multivariate analysis model that used OPG added age and gender. The second model used the same variables as the first one and included the presence of established CVD and diabetes. The third model used all previous variables and included an inflammatory activity marker, C-reactive protein (hsCRP). The final model included all the aforementioned variables, and patients CKD stages. The normality of the variables was evaluated using the Kolmogorov-Smirnov test. $p$ values $<0.05$ were considered statistically significant. Data were analyzed using Stata/SE v. 14.1 (StataCorp LP, College Station, TX, USA).

\section{Results}

Baseline characteristics. Altogether, 145 (89 males) patients, including 54 non-dialysis patients with CKD stages 3-5, 36 hemodialysis (HD) patients, and 55 peritoneal dialysis patients, fulfilled the study eligibility criteria and agreed to participate in the study. All dialysis patients did not present residual diuresis. Of the patients under conservative treatment, 9 started dialysis therapy during the period observed. The median followup was 36,5 months, the minimum 0,4 month and the maximum 66 months.

The OPG cutoff value determined using the ROC curve for all causes mortality was $10 \mathrm{pmol} / \mathrm{L}$ (area under de curve [AUC] 0.81; CI 95\%: 0.73-0.88; $p<0.001$ ) and $10.08 \mathrm{pmol} / \mathrm{L}$ for CV mortality (AUC 0.75; CI 95\%: $0.65-0.85, p<0.001$ ) (Fig. 1). 
A

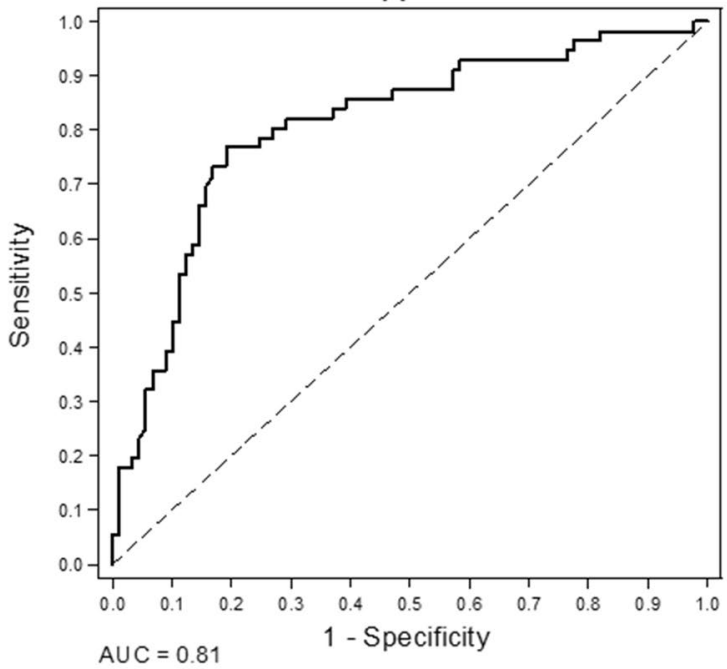

B

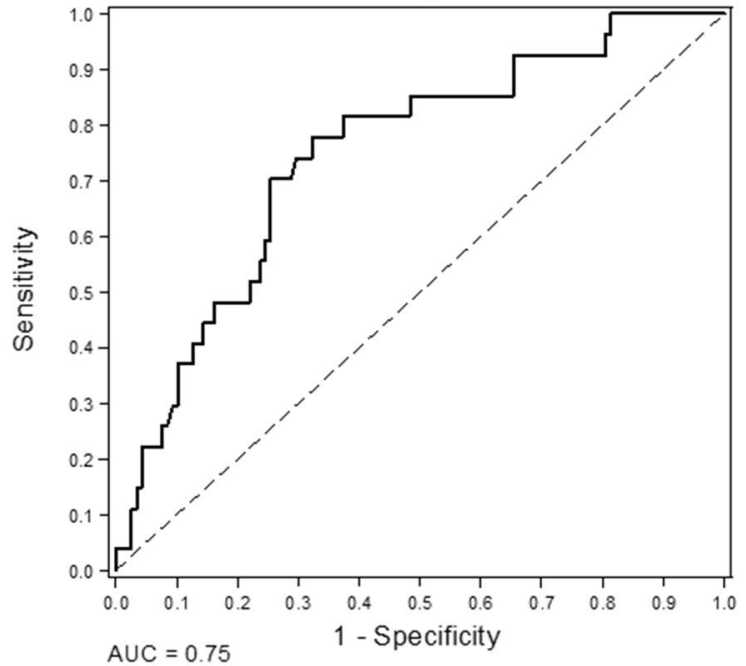

Figure 1. ROC curves for OPG-(A) All causes mortality (AUC 0.81, CI 95\%: 0.73-0.88); (B) CV mortality (AUC: 0.75; CI 95\%: 0.65-0.85).

Clinical and biochemical characteristics of the 145 patients included in the study are summarized in Tables 1 and 2. The levels of OPG for the whole cohort had a median value of 8.91 (25-75th percentiles 6.51-12.43) $\mathrm{pmol} / \mathrm{L}$.

Univariate correlations, assessed using Spearman's rank, demonstrated that OPG levels were significantly associated with age $(\mathrm{r}=0.37, p<0.01)$, left ventricle mass index $(\mathrm{r}=0.23, p=0.007)$, S100 calcium-binding protein A12 (S100A12) $(\mathrm{r}=0.21, p=0.010)$, receptor for advanced glycation end products soluble form (sRAGE) $(\mathrm{r}=0.33$, $p<0.001)$, IL-6 $(\mathrm{r}=0.39, p<0.001)$, FGF-23 $(\mathrm{r}=0.26, p=0.001)$, hsCRP $(\mathrm{r}=0.25, p=0.003)$, troponin I $(\mathrm{r}=0.54$, $p<0.001)$, IMT $(\mathrm{r}=0.40, p<0.001)$, and ejection fraction, $\mathrm{EF}(\mathrm{r}=-0.22, p=0.010)$. However, when a multivariate analysis was performed, only age $(p<0.001)$, fibroblast growth factor-23 ( $p=0.005)$, IL-6 $(p=0.017)$, troponin I $(p=0.002)$ and sRAGE $(p=0.002)$ were independently correlated with OPG levels.

OPG levels and clinical outcome. Univariate analysis of mortality showed that elevated OPG levels $(>10 \mathrm{pg} / \mathrm{mL}$ for general causes death and $>10.08 \mathrm{pg} / \mathrm{mL}$ for CV death) were related to higher mortality for both general and cardiovascular causes (SHR 6.81; CI 95\%: 3.69-12.6, $p<0.001$ and SHR 5.47; CI 95\%: 2.24-13.4, $p<0.001$, respectively), together with levels of serum troponin (SHR $=7.73$, CI 95\%: 3.17-18.8, $p<0.001$ and SHR 10.8, CI 95\%: 4.79-22.8), $p<0.001$, respectively) and $\log$ (sRAGE) levels (SHR=1.47, CI 95\%: 0.87-2.49, $p=0.150$ and SHR 2.07; CI 95\%: 1.07-3.95, $p=0.035$, respectively), and treatment by dialysis (SHR 4.80, CI 95\%: 2.17-10.6, $p<0.001$ and SHR 8.73, CI 95\%: 1.86-32.5, $p=0.003$, respectively).

Aalen-Johansen cumulative incidence curves showed a higher mortality for general (Fig. 2) and cardiovascular (Fig. 3) causes in the group with elevated OPG (>10 pmol/L for general causes and $>10.08 \mathrm{pmol} / \mathrm{L}$ for CV causes) early in the follow-up period, with an increase in the gap over time (during the follow-up period, 25 patients had undergone kidney transplantation and 16 were lost to follow-up, of which 9 were in the non-dialysis group). The number of deaths was 56 (cardiovascular: 25; infection: 17; malignances: 4; and other causes: 10).

In multivariate analysis, in a model including gender and age, OPG was found to be an independent marker of all-cause and cardiovascular mortality. When presence of previous CVD and diabetes, and hsCRP, were added, the results were similar. However, when CKD stages were included in the analysis, OPG was an independent marker only for all-cause mortality. (Tables 3,4 ).

\section{Discussion}

The main cause of death in our cohort was cardiovascular diseases, accounting for $45 \%$ (25 cases) of all deaths. This result is in line with those reported in the current literature, wherein large population studies have shown a progressive increase in the incidence of cardiovascular mortality with worsening renal function ${ }^{17}$.

The main finding in the present study was that OPG was a marker of higher cardiovascular mortality in patients with CKD, regardless of gender and age and other cardiovascular risk factors such as presence of diabetes, previous history of CVD as well as inflammation. We have been previously reported that an increased OPG concentration was associated with increased all-cause 3-year mortality risk among patients with CKD stages $3-5^{13}$. In the current study, the follow-up of this cohort was extended to 5, 5 years and, in addition to all-cause mortality risk, we also analyzed the association of OPG with cardiovascular mortality. Furthermore, the quality of the analysis was improved as we applied a ROC derived cut-off level of OPG in the survival analyses. The present results corroborate the results of several other studies, including those by Morena et al. and Matsubara et al. who found higher mortality in dialysis patients with high OPG levels ${ }^{18,19}$. Recently, Alderson et al. reported that OPG levels were associated with increased mortality risk in the CKD population ${ }^{8}$. Kuźniewski et al. proposed that the relationship between OPG and tumor necrosis factor-related apoptosis-inducing ligand (TRAIL) would act as a 


\begin{tabular}{|c|c|c|c|}
\hline & $\mathrm{OPG} \leq 10.08 \mathrm{pmol} / \mathrm{L}(\mathrm{n}=85)$ & OPG $>10.08 \mathrm{pmol} / \mathrm{L}(\mathrm{n}=60)$ & $p$ value $^{*}$ \\
\hline Age (years) & $55.0 \pm 15.2$ & $64.4 \pm 13.0$ & $<0.001$ \\
\hline Gender (male) & $57(67.1)$ & $32(53.3)$ & 0.119 \\
\hline Diabetes (yes) & $23(27.1)$ & $20(33.3)$ & 0.462 \\
\hline Dialysis (yes) & $36(42.4)$ & $55(91.7)$ & $<0.001$ \\
\hline Hemodalysis (yes) & $19(22)$ & $17(28)$ & 0.439 \\
\hline Peritoneal Dialysis (yes) & $17(20)$ & $38(63)$ & $<0.001$ \\
\hline CVD & $14(16.5)$ & $17(28.3)$ & 0.102 \\
\hline $\mathrm{Hb}(\mathrm{g} / \mathrm{dL})$ & $12.3 \pm 2.0$ & $11.1 \pm 2.1$ & 0.001 \\
\hline Albumin (g/dL) & $4.2 \pm 0.6$ & $3.7 \pm 0.6$ & 0.001 \\
\hline $\mathrm{P}(\mathrm{mg} / \mathrm{dL})$ & $4.5 \pm 1.7$ & $4.8 \pm 1.6$ & 0.351 \\
\hline hsCRP (mg/dL) & $5.5 \pm 7.6$ & $10.6 \pm 14.7$ & 0.017 \\
\hline IL-6 (pg/mL) & $5.7 \pm 11.9$ & $10.4 \pm 13.6$ & $<0.001$ \\
\hline TNF-a (pg/mL) & $16.9 \pm 6.1$ & $19.5 \pm 9.3$ & 0.063 \\
\hline PTX3 (pg/mL) & $4.0 \pm 2.4$ & $6.1 \pm 7$ & 0.001 \\
\hline Fetuin $(\mathrm{pg} / \mathrm{mL})$ & $0.43 \pm 0.10$ & $0.40 \pm 0.11$ & 0.109 \\
\hline FGF-23 (pg/mL) & $4273 \pm 11,106$ & $5013 \pm 12,023$ & 0.019 \\
\hline S100A (ng/mL) & $63.6 \pm 74.4$ & $101.8 \pm 96.3$ & 0.008 \\
\hline sRAGE (pg/mL) & $1981 \pm 1214$ & $2577 \pm 1124$ & $<0.001$ \\
\hline Troponin I (ng/mL) & $0.07 \pm 0.07$ & $0.19 \pm 0.29$ & $<0.001$ \\
\hline Diastolic dysfunction & $52(61.2)$ & $47(78.3)$ & 0.031 \\
\hline Ejection fraction $>45 \%$ & $76(93.8)$ & $50(89.3)$ & 0.356 \\
\hline LV mass index $\left(\mathrm{g} / \mathrm{m}^{2}\right)$ & $58.1 \pm 16.6$ & $68.0 \pm 23.9$ & 0.008 \\
\hline IMT mean $(\mathrm{cm})$ & $0.67 \pm 0.21$ & $0.85 \pm 0.42$ & 0.001 \\
\hline Death (all-cause) & $13(15.3)$ & $43(71.7)$ & $<0.001$ \\
\hline CV death & $6(7.1)$ & $21(35.0)$ & $<0.001$ \\
\hline
\end{tabular}

Table 1. Clinical and biochemical characteristics according to OPG levels. *Fisher exact test or Student's $\mathrm{t}$ test; data of non-normal continuous variables were submitted to a logarithmic transformation; $p<0.05$. Quantitative variables are expressed as mean \pm standard deviation. Categorical variables are expressed as frequencies (percent). OPG, osteoprotegerin; Hb, hemoglobin; P, phosphate; hsCRP, high-sensitivity C-reactive protein; IL-6, interleukin-6; TNF-a, tumor necrosis factor alpha; PTX3, pentraxin-related protein; FGF-23, fibroblast growth factor 23; sRAGE, receptor for advanced glycation end products (RAGE) soluble form; LV, left ventricle; IMT, intima-media thickness; CV, cardiovascular.

marker of increased cardiovascular mortality risk in stage 5 CKD patients, and, in their study, isolated OPG values were also able to predict all-cause and cardiovascular mortality ${ }^{20}$. In a recent meta-analysis Huang et al. found a relationship between OPG and increased cardiovascular mortality in patients with CKD, however, cited as some limitations: most studies including only dialysis patients, lack of adjustment for potential confounders and the lack of several studies in the area ${ }^{21}$. Altogether these studies suggest that an elevated circulating concentration of OPG appears to be a marker for increased all-cause and cardiovascular mortality risk in patients with CKD.

Although OPG is classically associated with vascular calcification, the exact mechanisms by which OPG may lead to higher mortality are still unknown. One of the current theories is that OPG is also a marker of atherosclerotic disease and myocardial ischemia ${ }^{22-26}$. Our results demonstrated that circulating OPG levels were directly correlated with serum troponin levels, one of the main markers of myocardial damage, corroborating these data.

Regarding cardiovascular disease, another possible question would be the relationship between OPG and ventricular function, since it is possible that OPG can also be secreted by the heart ${ }^{27}$. While previous studies were divergent regarding this relationship, Lindberg et al. found a correlation between OPG levels and a lower ejection fraction in patients after an acute ischemic event in a study including 42 patients. However, Shetelig et al. did not find this relationship in 272 patients with coronary disease ${ }^{28,29}$. While our study found no independent correlation between OPG and ejection fraction, diastolic dysfunction, or increased ventricular mass, OPG was a marker of increased mortality independent of the presence of previously known heart disease in patients with $\mathrm{CKD}$, when this and other well established risk factors (age, sex, diabetes, and inflammation) were included in a multivariate analysis.

Some authors propose that high OPG levels may be a direct consequence of vascular inflammation ${ }^{19}$. Other studies have demonstrated that OPG expression can be induced by the vascular musculature through inflammatory cytokines, reinforcing this idea ${ }^{30,31}$. However, our study did not find a correlation between inflammatory markers and OPG, and when an inflammation marker (hsCRP) was included in the multivariate analysis, OPG remained as an independent marker of cardiovascular mortality. Sigrist et al. reported similar results, in that high levels of OPG were associated with higher mortality independently of hsCRP levels in patients with CKD ${ }^{32}$.

Recently, Wieczorek-Surdacka et al. ${ }^{33}$ reported that elevated levels of OPG were associated with a worse renal and cardiovascular outcome in patients with chronic renal disease and stable coronary artery disease and that 


\begin{tabular}{|c|c|c|c|c|}
\hline & \multicolumn{3}{|l|}{ CKD } & \multirow[b]{2}{*}{$p^{*}$} \\
\hline & Stage $3(n=26 ; 17.9 \%)$ & Stage $4(n=30 ; 20.7 \%)$ & Stage $5(n=89 ; 61.4 \%)$ & \\
\hline Age (years) & $61.8 \pm 11$ & $62.2 \pm 14.3$ & $57.0 \pm 16.0$ & 0.163 \\
\hline Gender (male) & $16(61.5)$ & $21(70)$ & $52(58.4)$ & 0.530 \\
\hline Diabetes (yes) & 7 (26.9) & $13(43.3)$ & $23(25.8)$ & 0.182 \\
\hline OPG pmol/L & $6.3 \pm 2.2^{\mathrm{a}}$ & $7.8 \pm 3.2^{\mathrm{a}}$ & $11.9 \pm 5.8^{\mathrm{b}}$ & $<0.001$ \\
\hline $\mathrm{OPG}>10 \mathrm{pmol} / \mathrm{L}$ & $2(7.7)^{\mathrm{a}}$ & $5(16.7)^{\mathrm{a}}$ & $53(59.6)^{b}$ & $<0.001$ \\
\hline CVD & $4(15.4)$ & $5(16.7)$ & $22(24.7)$ & 0.462 \\
\hline $\mathrm{Hb}(\mathrm{g} / \mathrm{dL})$ & $13.2 \pm 1.7^{\mathrm{a}}$ & $12.9 \pm 2.1^{\mathrm{a}}$ & $11.1 \pm 1.9^{\mathrm{b}}$ & $<0.001$ \\
\hline Albumin (g/dL) & $4.4 \pm 0.2$ & $4.1 \pm 0.9$ & $3.8 \pm 0.6$ & 0.083 \\
\hline $\mathrm{P}(\mathrm{mg} / \mathrm{dL})$ & $3.4 \pm 0.8^{\mathrm{a}}$ & $3.7 \pm 0.8^{\mathrm{a}}$ & $5.2 \pm 1.7^{\mathrm{b}}$ & $<0.001$ \\
\hline hsCRP (mg/dL) & $6.4 \pm 10.9$ & $7.2 \pm 14.4$ & $8.1 \pm 10.4$ & 0.099 \\
\hline IL-6 (pg/mL) & $3.3 \pm 4.7^{\mathrm{a}}$ & $9.3 \pm 18.6^{\mathrm{b}}$ & $8.3 \pm 11.8^{b}$ & $<0.001$ \\
\hline TNF-a (pg/mL) & $14.8 \pm 5.5^{\mathrm{a}}$ & $15.6 \pm 8.6^{\mathrm{a}}$ & $19.6 \pm 7.4^{\mathrm{b}}$ & 0.003 \\
\hline PTX3 (pg/mL) & $3.2 \pm 1.6^{\mathrm{a}}$ & $3.6 \pm 2.1^{\mathrm{a}}$ & $5.8 \pm 3.7^{\mathrm{b}}$ & $<0.001$ \\
\hline Fetuin (pg/mL) & $0.43 \pm 0.09$ & $0.44 \pm 0.07$ & $0.41 \pm 0.11$ & 0.283 \\
\hline FGF-23 (pg/mL) & $154 \pm 103^{\mathrm{a}}$ & $192 \pm 196^{\mathrm{a}}$ & $7386 \pm 13,981^{b}$ & $<0.001$ \\
\hline S100A (ng/mL) & $59.7 \pm 74.1$ & $52.9 \pm 34.1$ & $93.9 \pm 97.6$ & 0.166 \\
\hline sRAGE (pg/mL) & $1539 \pm 876^{\mathrm{a}}$ & $1710 \pm 1053^{\mathrm{a}}$ & $2600 \pm 1200^{\mathrm{b}}$ & $<0.001$ \\
\hline Troponin I (ng/mL) & $0.05 \pm 0.04^{\mathrm{a}}$ & $0.10 \pm 0.11^{\mathrm{a}}$ & $0.15 \pm 0.25^{\mathrm{b}}$ & $<0.001$ \\
\hline Diastolic dysfunction & $21(80.8)$ & $24(80)$ & $54(60.7)$ & 0.050 \\
\hline Ejection fraction $>45 \%$ & $2(8.3)$ & $2(6.9)$ & $7(8.3)$ & 0.969 \\
\hline LV mass index $\left(\mathrm{g} / \mathrm{m}^{2}\right)$ & $62.5 \pm 17.2$ & $65.6 \pm 25.6$ & $60.6 \pm 19.2$ & 0.620 \\
\hline IMT mean $(\mathrm{cm})$ & $0.67 \pm 0.22$ & $0.72 \pm 0.25$ & $0.78 \pm 0.37$ & 0.379 \\
\hline Death (all-cause) & $5(19.2)^{\mathrm{a}}$ & $4(13.3)^{\mathrm{a}}$ & $47(52.8)^{\mathrm{b}}$ & $<0.001$ \\
\hline CV death & $1(3.8)^{\mathrm{a}}$ & $2(6.7)^{\mathrm{a}}$ & $24(27.0)^{\mathrm{b}}$ & 0.005 \\
\hline
\end{tabular}

Table 2. Clinical and biochemical characteristics according to CKD stage. ${ }^{\star}$ Chi-square test or one-way ANOVA; data of non-normal continuous variables were submitted to a logarithmic transformation; $p<0.05$. ${ }^{\mathrm{a}, \mathrm{b}}$ Different letters indicate significant difference $(p<0.05)$. Quantitative variables are expressed as mean \pm standard deviation. Categorical variables are expressed as frequencies (percent). OPG, osteoprotegerin; $\mathrm{Hb}$, hemoglobin; P, phosphate; hsCRP, high-sensitivity C-reactive protein; IL-6, interleukin-6; TNF-a, tumor necrosis factor alpha; PTX3, pentraxin-related protein; FGF-23, fibroblast growth factor 23; sRAGE, receptor for advanced glycation end products (RAGE) soluble form; LV, left ventricle; IMT, intima-media thickness; CV, cardiovascular.

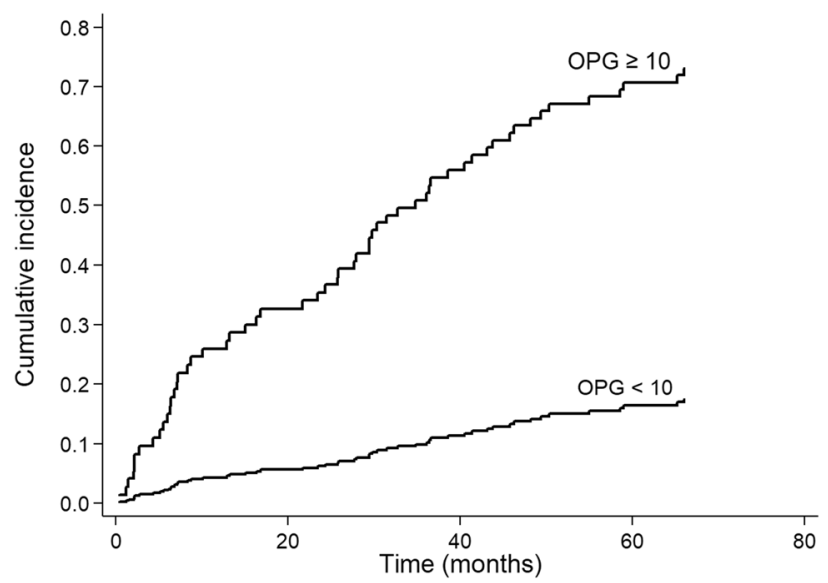

Figure 2. Aalen-Johansen cumulative incidence curves for all-cause mortality in $145 \mathrm{CKD}$ patients with OPG levels $\leq 10 \mathrm{pmol} / \mathrm{L}$ or $>10 \mathrm{pmol} / \mathrm{L}$. 


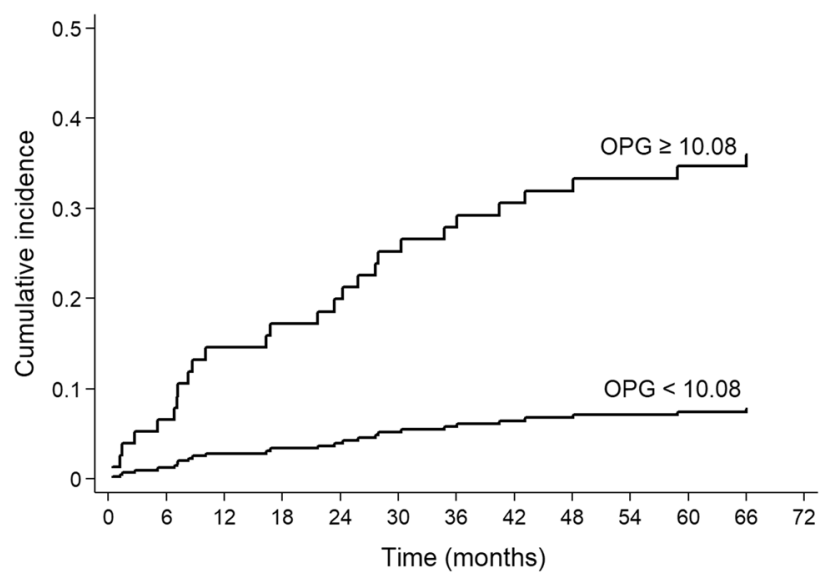

Figure 3. Aalen-Johansen cumulative incidence curves for cardiovascular death in $145 \mathrm{CKD}$ patients with OPG levels $\leq 10.08 \mathrm{pmol} / \mathrm{L}$ or $>10.08 \mathrm{pmol} / \mathrm{L}$.

\begin{tabular}{|c|c|c|c|c|}
\hline Model & Variable & $p$ value $^{\mathrm{a}}$ & SHR & CI 95\% \\
\hline Crude model & OPG & $<0.001$ & 1.10 & $1.06-1.14$ \\
\hline \multirow{3}{*}{ Model 1} & OPG & $<0.001$ & 1.08 & $1.05-1.12$ \\
\hline & Age & 0.079 & 1.02 & $1.00-1.05$ \\
\hline & Sex (male) & 0.145 & 0.68 & $0.40-1.14$ \\
\hline \multirow{5}{*}{ Model 2} & OPG & $<0.001$ & 1.08 & $1.05-1.12$ \\
\hline & Age & 0.128 & 1.02 & $1.00-1.04$ \\
\hline & Sex (male) & 0.109 & 0.64 & $0.40-1.10$ \\
\hline & Diabetes & 0.654 & 0.87 & $0.48-1.58$ \\
\hline & CVD & 0.277 & 1.44 & $0.74-2.79$ \\
\hline \multirow{6}{*}{ Model 3} & OPG & $<0.001$ & 1.08 & $1.04-1.12$ \\
\hline & Age & 0.121 & 1.02 & $1.00-1.05$ \\
\hline & Sex (male) & 0.089 & 0.62 & $0.36-1.08$ \\
\hline & Diabetes & 0.823 & 0.93 & $0.50-1.72$ \\
\hline & CVD & 0.371 & 1.40 & $0.68-2.85$ \\
\hline & hsCRP & 0.179 & 1.01 & $0.99-1.03$ \\
\hline \multirow{10}{*}{ Model 4} & OPG & 0.048 & 1.04 & $1.00-1.08$ \\
\hline & Age & 0.005 & 1.04 & $1.01-1.07$ \\
\hline & Sex (male) & 0.389 & 0.78 & $0.45-1.37$ \\
\hline & Diabetes & 0.816 & 0.93 & $0.49-1.74$ \\
\hline & CVD & 0.643 & 1.18 & $0.59-2.36$ \\
\hline & hsCRP & 0.029 & 1.02 & $1.00-1.04$ \\
\hline & \multicolumn{4}{|l|}{ CKD stage } \\
\hline & $3(\text { ref })^{\mathrm{a}}$ & & & \\
\hline & 4 & 0.164 & 0.35 & $0.08-1.53$ \\
\hline & 5 & 0.061 & 2.55 & $0.96-6.79$ \\
\hline
\end{tabular}

Table 3. Multivariate analysis for all-cause mortality. Fine and Gray model for mortality (any cause) considering transplant as competing risk. ${ }^{\text {a Stage }} 3$ used as a reference for the analysis. OPG, osteoprotegerin; CVD, cardiovascular disease; hsCRP, high-sensitivity C-reactive protein; CKD, Chronic Kidney Disease; SHR: subdistribution hazard ratio.

there was an independent relationship between elevated levels of OPG and low homoarginine (hArg)/symmetric dimethylarginine ratio (ADMA). However, our study did not include measurements of hArg or serum ADMA.

When CKD stages were included in the multivariate analysis, OPG was an independent marker of all-cause mortality but not cardiovascular mortality. This may to some extent reflect the link between elevated OPG levels and poor renal function ${ }^{34}$, and by the fact that cardiovascular mortality is increased in patients CKD stage 5 , especially in those undergoing dialysis ${ }^{35}$. 


\begin{tabular}{|c|c|c|c|c|}
\hline Model & Variable & $p$ value $^{\mathrm{a}}$ & SHR & CI 95\% \\
\hline Crude model & OPG & $<0.001$ & 1.10 & $1.05-1.15$ \\
\hline \multirow{3}{*}{ Model 1} & OPG & $<0.001$ & 1.10 & $1.04-1.15$ \\
\hline & Age & 0.855 & 1.00 & $0.97-1.03$ \\
\hline & Sex (male) & 0.879 & 0.94 & $0.44-2.02$ \\
\hline \multirow{5}{*}{ Model 2} & OPG & $<0.001$ & 1.10 & $1.04-1.16$ \\
\hline & Age & 0.929 & 1.00 & $0.97-1.03$ \\
\hline & Sex (male) & 0.833 & 0.92 & $0.43-1.99$ \\
\hline & Diabetes & 0.783 & 1.12 & $0.50-2.53$ \\
\hline & CVD & 0.426 & 1.43 & $0.60-3.42$ \\
\hline \multirow{6}{*}{ Model 3} & OPG & 0.001 & 1.10 & $1.04-1.16$ \\
\hline & Age & 0.757 & 1.00 & $0.96-1.03$ \\
\hline & Sex (male) & 0.904 & 1.05 & $0.48-2.30$ \\
\hline & Diabetes & 0.687 & 1.18 & $0.52-2.68$ \\
\hline & CVD & 0.340 & 1.53 & $0.64-3.66$ \\
\hline & hsCRP & 0.885 & 1.00 & $0.96-1.04$ \\
\hline \multirow{10}{*}{ Model 4} & OPG & 0.074 & 1.06 & $0.99-1.13$ \\
\hline & Age & 0.668 & 1.01 & $0.97-1.04$ \\
\hline & Sex (male) & 0.668 & 1.19 & $0.54-2.64$ \\
\hline & Diabetes & 0.616 & 1.24 & $0.54-2.83$ \\
\hline & CVD & 0.588 & 1.28 & $0.52-3.15$ \\
\hline & hsCRP & 0.869 & 1.00 & $0.96-1.04$ \\
\hline & \multicolumn{4}{|l|}{ CKD stage } \\
\hline & $3(\mathrm{ref})^{\star}$ & & & \\
\hline & 4 & 0.664 & 1.72 & $0.15-19.6$ \\
\hline & 5 & 0.105 & 5.34 & $0.71-40.4$ \\
\hline
\end{tabular}

Table 4. Multivariate analysis for cardiovascular-related mortality. Fine and Gray model for mortality (cardiovascular causes) considering transplant and other cause of mortality as competing risk. ${ }^{\text {a Stage }} 3$ used as a reference for the analysis OPG, osteoprotegerin; CVD, cardiovascular disease; hsCRP, high-sensitivity $\mathrm{C}$-reactive protein; $\mathrm{CKD}$, chronic kidney disease; SHR: subdistribution hazard ratio.

\section{Conclusions}

This study has some limitations that should be mentioned. First, this was an observational study, precluding any conclusions regarding causality. Second, the number of patients was relatively small, which did not allow us to include it in a specific multivariate analysis of mortality within non-dialysis group. Furthermore, only a single sample was collected, and the analysis was restricted to a certain time point, which may fail to reflect the natural course of the processes being studied. In addition, we did not measure other relevant markers that are associated with OPG and its actions such as RANKL and TRAIL or presence of vascular calcification asssed by coronary artery calcium score. Also, we do not have albuminuria measured in all cohort because most part of our dialysis patients did not have any residual function. Moreover, the lack of laboratory standardization to analyze albuminuria in a single urine sample made this analysis unfeasible. It would be necessary to collect $24 \mathrm{~h}$ urine samples which was not possible to perform in the present study.

In summary, we report that a high circulating OPG level was a prognostic indicator of increased all-cause and cardiovascular mortality risk in patients with CKD. These results suggest that OPG could be potentially useful in the prognostic evaluation of patients with $\mathrm{CKD}$, as previously proposed by other authors. Future research should investigate correlations between OPG and other markers of cardiovascular mortality, such as the coronary artery calcium score, and explore whether therapies aimed at reducing the high circulating concentration of OPG in patients with CKD may reduce the high cardiovascular mortality in this patient population.

\section{Data availability}

The datasets used and/or analyzed during the current study are available from the corresponding author on reasonable request.

Received: 18 April 2020; Accepted: 14 January 2021

Published online: 28 January 2021

\section{References}

1. Benjamin, E. J. et al. Heart disease and stroke statistics-2017 update: a report from the American Heart Association. Circulation 135, e146-e603 (2017).

2. Tonelli, M. et al. Chronic kidney disease and mortality risk: a systematic review. J. Am. Soc. Nephrol. 17, 2034-2047 (2006). 
3. Kundhal, K. \& Lok, C. E. Clinical epidemiology of cardiovascular disease in chronic kidney disease. Nephron. Clin. Pract. 101, c47-52 (2005).

4. Foley, R. N. Clinical epidemiology of cardiovascular disease in chronic kidney disease. J. Ren. Care. 36, 4-8 (2010).

5. Barreto, D. V. et al. Plasma interleukin- 6 is independently associated with mortality in both hemodialysis and pre-dialysis patients with chronic kidney disease. Kidney Int. 77, 550-556 (2010).

6. Nakashima, A. et al. Effect of circulating soluble receptor for advanced glycation end products (sRAGE) and the proinflammatory RAGE ligand (EN-RAGE, S100A12) on mortality in hemodialysis patients. Clin. J. Am. Soc. Nephrol. 5, 2213-2219 (2010).

7. Lee, B. T. et al. Association of C-reactive protein, tumor necrosis factor-alpha, and interleukin-6 with chronic kidney disease. BMC Nephrol. 16, 77 (2015).

8. Alderson, H. V. et al. FGF-23 and Osteoprotegerin but not Fetuin-A are associated with death and enhance risk prediction in non-dialysis chronic kidney disease stages 3-5. Nephrology 21, 566-573 (2016).

9. Mesquita, M. et al. Plasma osteoprotegerin is an independent risk factor for mortality and an early biomarker of coronary vascular calcification in chronic kidney disease. Clin. Chem. Lab. Med. 47, 339-346 (2009).

10. Mesquita, M. et al. Osteoprotegerin and progression of coronary and aortic calcifications in chronic kidney disease. Transplant. Proc. 42, 3444-3449 (2010).

11. Tschiderer, L., Willeit, J., Schett, G., Kiechl, S. \& Willeit, P. Osteoprotegerin concentration and risk of cardiovascular outcomes in nine general population studies: literature-based meta-analysis involving 26,442 participants. PLoS ONE 12, e0183910 (2017).

12. Nakashima, A. et al. Plasma osteoprotegerin, arterial stiffness, and mortality in normoalbuminemic Japanese hemodialysis patients. Osteoporos. Int. 22, 1695-1701 (2011).

13. Nascimento, M. M., Hayashi, S. Y., Riella, M. C. \& Lindholm, B. Elevated levels of plasma osteoprotegerin are associated with allcause mortality risk and atherosclerosis in patients with stages 3-5 chronic kidney disease. Braz. J. Med. Biol. Res. 47, 995-1002 (2014).

14. Stein, J. H. et al. Use of carotid ultrasound to identify subclinical vascular disease and evaluate cardiovascular disease risk: a consensus statement from the American Society of Echocardiography Carotid Intima-Media Thickness Task Force endorsed by the Society for Vascular Medicine. J. Am. Soc. Echocardiogr. 21, 93-111 (2008).

15. Quiñones, M. A., Otto, C. M., Stoddard, M., Waggoner, A. \& Zoghbi, W. A. Recommendations for quantification of Doppler echocardiography: a report from the Doppler Quantification Task Force of the Nomenclature and Standards Committee of the American Society of Echocardiography. J. Am. Soc. Echocardiogr. 15, 167-184 (2002).

16. Lang, R. M. et al. Recommendations for chamber quantification: a report from the American Society of Echocardiography's Guidelines and Standards Committee and the Chamber Quantification Writing Group, developed in conjunction with the European Association of Echocardiography, a branch of the European Society of Cardiology. J. Am. Soc. Echocardiogr. 18, 1440-1463 (2005).

17. Thompson, S. et al. Cause of death in patients with reduced kidney function. J. Am. Soc. Nephrol. 26, 2504-2511 (2015).

18. Morena, M. et al. Plasma osteoprotegerin is associated with mortality in hemodialysis patients. J. Am. Soc. Nephrol. 17, 262-270 (2006).

19. Matsubara, K. et al. Inflammation modifies the association of osteoprotegerin with mortality in chronic kidney disease. J. Nephrol. 11, 774 (2009).

20. Kuźniewski, M. et al. Osteoprotegerin and osteoprotegerin/TRAIL ratio are associated with cardiovascular dysfunction and mortality among patients with renal failure. Adv. Med. Sci. 61, 269-275 (2016).

21. Huang, Q. et al. Elevated osteoprotegerin concentration predicts increased risk of cardiovascular mortality in patients with chronic kidney disease: a systematic review and meta-analysis. Kidney Blood Press Res. 45, 565-575 (2020).

22. Omland, T. et al. Plasma osteoprotegerin levels in the general population: relation to indices of left ventricular structure and function. Hypertension 49, 1392-1398 (2007).

23. Abedin, M. et al. Relation of osteoprotegerin to coronary calcium and aortic plaque (from the Dallas Heart Study). Am. J. Cardiol. 99, 513-518 (2007).

24. Yilmaz, M. I. et al. Osteoprotegerin in chronic kidney disease: associations with vascular damage and cardiovascular events. Calcif. Tissue. Int. 99, 121-130 (2016).

25. Pateinakis, P., Papagianni, A., Douma, S., Efstratiadis, G. \& Memmos, D. Associations of fetuin-A and osteoprotegerin with arterial stiffness and early atherosclerosis in chronic hemodialysis patients. BMC Nephrol. 14, 122 (2013).

26. Shaker, O. G., El-Shehaby, A. \& Nabih, M. Possible role of osteoprotegerin and tumor necrosis factor-related apoptosis-inducing ligand as markers of plaque instability in coronary artery disease. Angiology 61, 756-762 (2010).

27. Schoppet, M. et al. Increased osteoprotegerin serum levels in men with coronary artery disease. J. Clin. Endocrinol. Metab. 88, 1024-1028 (2003).

28. Shetelig, C. et al. Osteoprotegerin levels in ST-elevation myocardial infarction: temporal profile and association with myocardial injury and left ventricular function. PLoS ONE 12, e0173034 (2017).

29. Lindberg, S. et al. Osteoprotegerin levels change during STEMI and reflect cardiac function. Can. J. Cardiol. 30, 1523-1528 (2014).

30. Zhang, J. et al. PDGF induces osteoprotegerin expression in vascular smooth muscle cells by multiple signal pathways. FEBS Lett. 521, 180-184 (2002).

31. Raaz-Schrauder, D. et al. Plasma levels of sRANKL and OPG are associated with atherogenic cytokines in patients with intermediate cardiovascular risk. Heart Vessels 32, 1304-1313 (2017).

32. Sigrist, M. K., Levin, A., Er, L. \& McIntyre, C. W. Elevated osteoprotegerin is associated with all-cause mortality in CKD stage 4 and 5 patients in addition to vascular calcification. Nephrol. Dial. Transplant. 24, 3157-3162 (2009).

33. Wieczorek-Surdacka, E. et al. Distinct associations between plasma osteoprotegerin, homoarginine and asymmetric dimethylarginine in chronic kidney disease male patients with coronary artery disease. Amino Acids 51, 977-982 (2019).

34. Vik, A. et al. Serum osteoprotegerin and renal function in the general population: the Tromso Study. Clin. Kidney J. 10, 38-44 (2017).

35. Go, A. S., Chertow, G. M., Fan, D., McCulloch, C. E. \& Hsu, C. Y. Chronic kidney disease and the risks of death, cardiovascular events, and hospitalization. N. Engl. J. Med. 351, 1296-1305 (2004).

\section{Acknowledgements}

We would like to thank the patients and personnel involved in the creation of this cohort, our research staff at the clinical research center, and Britta Lind for laboratory assessments.

\section{Author contributions}

G.L.M. contributed in data review, interpretation of results and manuscript writing.S.H. contributed in cardiovascular and echocardiogram assessmentsA.B., M.L. and B.L. contributed in laboratory assessments, supervision and manuscript reviewM.R. and M.M.N. contributed to this work in design of study, patient follow-up and supervisionM.O. contributed in statistical analysis. 


\section{Funding}

There is no specific funding for this research involved in collection, analysis, interpretation of data and in writing of the manuscript . Baxter Novum is the result of a grant from Baxter Healthcare to Karolinska Institute.

\section{Competing interests}

Bengt Lindholm is employed by Baxter Healthcare. None of the other authors report any competing interests.

\section{Additional information}

Correspondence and requests for materials should be addressed to G.L.M.

Reprints and permissions information is available at www.nature.com/reprints.

Publisher's note Springer Nature remains neutral with regard to jurisdictional claims in published maps and institutional affiliations.

(c) (i) Open Access This article is licensed under a Creative Commons Attribution 4.0 International License, which permits use, sharing, adaptation, distribution and reproduction in any medium or format, as long as you give appropriate credit to the original author(s) and the source, provide a link to the Creative Commons licence, and indicate if changes were made. The images or other third party material in this article are included in the article's Creative Commons licence, unless indicated otherwise in a credit line to the material. If material is not included in the article's Creative Commons licence and your intended use is not permitted by statutory regulation or exceeds the permitted use, you will need to obtain permission directly from the copyright holder. To view a copy of this licence, visit http://creativecommons.org/licenses/by/4.0/.

(C) The Author(s) 2021 